\title{
PERFORMANCE OF SEMI-ORTHOGONAL USER SELECTION FOR MULTIUSER MISO SYSTEMS
}

Didier LE RUYET, Hajer KHANFIR

Electronics and Communications Laboratory, CNAM, 292 rue Saint Martin, 75141, Paris, France

leruyet@cnam.fr, hajer.khanfir@cnam.fr

\section{ABSTRACT}

In MISO multiuser downlink wireless communication systems with precoding at the transmission, the capacity depends on the channel state information at the transmitter. Since the feedback data rate increases with the number of users in the cell it is important to perform a selection of the users at the receiver. In this paper, we evaluate the performance of different user selection algorithms at the receiver side through the noisy uplink channel. We evaluate the effect of the noisy channel on the classical norm criterion and on a criterion based on the orthogonality between the user channels. Without cooperation between the users, we only allow users that are semi-orthogonal to feedback their channel information as analog and quantized information to the base station through the noisy uplink channel. We propose an algorithm to reduce the noise effect on the analog feedback for semi-orthogonal user selection algorithm. We show that the semi-orthogonal criterion with quantized feedback gives better performance compared to the norm criterion for perfect and noisy uplink channels.

\section{INTRODUCTION}

In a multi-user downlink system with $N_{t}$ antennas at the base station and single antenna receivers, the capacity is achieved when the base station transmits simultaneously to $N_{t}$ selected users using Dirty Paper Coding (DPC). When the number of users is much higher than $N_{t}$, an efficient user selection algorithm must be used to reduce the feedback data rate [1]. The selected users must send their channel quality information (CQI) and channel direction information (CDI) for the reduction of interference. Using the feedback information, the transmitter will select the best set of users and then perform precoding. In this paper, we propose to reduce the feedback information thanks to the selection of a group of semi-orthogonal users at the user side. In order to limit the feedback rate, we will consider both unquantized and quantized CDI. We will evaluate the performance of the system considering a noisy uplink channel. Finally, we will propose an algorithm to reduce the noise effect on the analog feedback for semi-orthogonal user selection algorithm.

This paper is organized as follows. Section 2 review briefly multiuser system model. Norm criterion and quasi orthogonal criterion are introduced in Section 3. Section 4 is devoted to the quantized and analog feedback link. Section 5 presents and discusses the simulation results and Section 6 draws the concluding remarks of this paper.
Berna ÖZBEK

Electrical and Electronics Engineering Department, Izmir Institute of Technology, Urla, 35430, Izmir,Turkey

bernaozbek@iyte.edu.tr

\section{SYSTEM MODEL}

We consider a multi-user downlink system with $N_{t}$ antennas at the base station and $K$ mobile users, each equipped with a single receive antenna. The received signal is corrupted by Additive White Gaussian Noise (AWGN). We suppose that all the users are independent and distributed in a homogeneous way: the average signal-to-noise ratio (SNR) is the same for all the receivers. We also assume that the channel is perfectly known at the receiver.

At each block, the received signal for $k$-th user can be written as:

$$
\begin{aligned}
y_{k} & =\mathbf{h}_{k}^{H} \mathbf{x}+n_{k} \quad k=1, \cdots, K \\
& =\mathbf{h}_{k}^{H} \mathbf{W P s}+n_{k}
\end{aligned}
$$

where $\mathbf{P}=\operatorname{diag}\left(\sqrt{P_{1}}, \cdots, \sqrt{P_{K}}\right)$, the matrix for power loading, $\mathbf{W}=\left[\mathbf{w}_{1} \cdots \mathbf{w}_{K}\right]$ is a precoding matrix (the columns of $\mathbf{W}$ are thus normalized to unit norm), $\mathbf{x} \in \mathcal{C}^{N_{t} \times 1}$ is the transmitted symbol from the base station antennas, subject to a short-term power constraint (the transmitted signal must satisfy power constraint $\mathbf{x}^{H} \mathbf{x} \leq P$ ) and we consider equal power allocation over each transmit beam, $\mathbf{h}_{k} \in \mathcal{C}^{N_{t} \times 1}$ is the channel gain vector to the $k$-th user with independently and identically distributed (i.i.d.) complex Gaussian entries and $\mathbf{s}$ is the data vector for the scheduled users at time $T$. The complex coefficients of the channel vector and noise $n_{k} \in \mathcal{C}$ are i.i.d and zero mean Gaussian variables. We normalize the channel such that the entries of $\mathbf{h}_{k}$ have unit variance.

For a system where $N_{t}<K$, let $\mathcal{S}$ be the set of $N_{t}$ users scheduled at time $T$. Then the associated users' data are transmitted via Zero-forcing Beamforming (ZFBF) precoding [2] by exploiting the channel state information at the transmitter (CSIT). Then $\mathbf{H}(\mathcal{S})$ denotes the matrix consisting of $N_{t}$ channel vectors of the selected users at time $T$. The relation between the data vector $\mathbf{s}(\mathcal{S})$ and the transmitted vector $\mathbf{x}(\mathcal{S})$ is given by:

$$
\mathbf{x}(\mathcal{S})=\mathbf{W}(\mathcal{S}) \mathbf{P s}(\mathcal{S})
$$

where $\mathbf{P}=\operatorname{diag}\left(\sqrt{\frac{P}{N_{t}}}, \cdots, \sqrt{\frac{P}{N_{t}}}\right)$, is the $N_{t} \times N_{t}$ matrix for uniform power loading. The $\mathrm{ZF}$ transmit beamforming vector is : $\mathbf{W}(\mathcal{S})=\alpha \mathbf{H}(\mathcal{S})^{H}\left(\mathbf{H}(\mathcal{S})^{H} \mathbf{H}(\mathcal{S})\right)^{-1}$

In order to keep the short term power constant we have:

$$
\alpha=\frac{1}{\sqrt{\operatorname{tr}\left(\left(\mathbf{H}(\mathcal{S})^{H} \mathbf{H}(\mathcal{S})\right)^{-1}\right)}}
$$

The sum rate achieved by the ZFBF scheme is: 


$$
R_{Z F B F}(\mathcal{S})=\max _{\mathbf{w}_{k}} \sum_{k \in \mathcal{S}} \log \left(1+S I N R_{k}\right)
$$

The signal-to-interference-plus-noise-ratio (SINR) for every user is:

$$
S I N R_{k}=\frac{\left|\mathbf{h}_{k}^{H} \mathbf{w}_{k}\right|^{2}}{\sigma^{2}+\sum_{j \neq k}^{N_{t}}\left|\mathbf{h}_{k}^{H} \mathbf{w}_{j}\right|^{2}}
$$

The achievable sum rate of $\mathrm{ZFBF}$ is found by considering every possible choice of user groups $\mathcal{S}$ :

$$
R_{Z F B F}=\max _{\mathcal{S} \subset\{1, \cdots, K\}:|\mathcal{S}|=N_{t}} R_{Z F B F}(\mathcal{S})
$$

\section{USER SELECTION CRITERIA}

In order to maximize the sum rate of the downlink system under an average power constraint $P$, the scheduler at the base station should choose the best combination of $N_{t}$ users. However, the exhaustive search which consists in evaluating $\left(\begin{array}{c}K \\ N_{t}\end{array}\right)$ combinations becomes quickly prohibitive. In this paper, we propose to reduce the number of selected users since users having a poor channel (low norm or/and interfering with other good users) will have no chance to be chosen. Consequently, they should not take part in the user selection algorithm, nor feedback their channel information.For the construction of the selected user group, we propose three self discrimination criteria.

\subsection{Norm-only criterion}

The norm-only criterion only selects users whose channel norms are above a threshold $\gamma_{t h}[1]$.

$$
\mathcal{T}_{1}=\left\{k \in \mathcal{R}:\left\|\mathbf{h}_{k}\right\|^{2}>\gamma_{t h}\right\}
$$

where $\mathcal{R}$ be the set of all users.

\subsection{Semi-orthogonal criterion}

The semi-orthogonal criterion selects users whose channel directions (CDI) are semi-orthogonal. Each user generates the same $N_{t}$ random orthonormal vectors $\phi_{i}\left(N_{t} \times 1\right), i=1, \ldots, N_{t}$. The users measure the orthogonality between their channels and the random vectors $\phi_{i}$ using the chordal distance:

$$
d^{2}\left(\overline{\mathbf{h}}_{k}, \phi_{i}\right)=1-\left|\overline{\mathbf{h}}_{k}^{H} \phi_{i}\right|^{2}
$$

where $\overline{\mathbf{h}}_{k}=\frac{\mathbf{h}_{k}}{\left\|\mathbf{h}_{k}\right\|}$ is the normalized channel vector of the user $k$.

Let $\mathcal{O}^{N_{t}}$ be the unit sphere lying in $\mathcal{C}^{N_{t}}$ and centered at the original. Using the chordal distance metric, for any $\epsilon_{t h}<1$, we can define a spherical cap on $\mathcal{O}^{N_{t}}$ with center $\boldsymbol{o}$ and square radius $\epsilon_{t h}$ as the open set :

$$
\mathcal{B}_{\epsilon}(\mathbf{o})=\left\{\mathbf{g} \in \mathcal{O}^{N_{t}}: d^{2}(\mathbf{g}, \mathbf{o}) \leq \epsilon_{t h}\right\}
$$

For the second criterion each user generates a set $\mathcal{F}=\left\{\phi_{1}, \ldots, \phi_{N t}\right.$ composed of $N_{t}$ unitary orthogonal vectors.

Then we have:

$$
\mathcal{T}_{2}=\left\{k \in \mathcal{R}: \overline{\mathbf{h}}_{k} \in \bigcup_{i=1}^{N_{t}} \mathcal{B}_{\epsilon}\left(\phi_{i}\right)\right\}
$$

where $\mathcal{T}_{2}$ is the set of near orthogonal users.

\subsection{Combined criterion}

The last criterion combines the two previous ones:

$$
\mathcal{T}_{3}=\left\{k \in \mathcal{R}: \overline{\mathbf{h}}_{k} \in \bigcup_{i=1}^{N_{t}} \mathcal{B}_{\epsilon}\left(\phi_{i}\right) \text { and }\left\|\mathbf{h}_{k}\right\|^{2}>\gamma_{t h}\right\}
$$

\subsection{Thresholds predetermination values}

We must choose the threshold values for the three sets in order to allow the number of average users $K_{a v g}$ to feedback their CDI and CQI.

The channel norm and its direction are independent and we have:

$$
\begin{aligned}
K_{\text {avg }} & =K \mathcal{P}\left\{k \in \mathcal{T}_{3}\right\} \\
& =K \mathcal{P}\left\{k \in \mathcal{T}_{1}\right\} \times \mathcal{P}\left\{k \in \mathcal{T}_{2}\right\} \\
& =K \mathcal{P}\left\{\left\|\mathbf{h}_{k}\right\|^{2}>\gamma_{t h}\right\} \times \mathcal{P}\left\{k \in \mathcal{R}: \overline{\mathbf{h}}_{k} \in \bigcup_{i=1}^{N_{t}} \mathcal{B}_{\epsilon}\left(\phi_{i}\right)\right\}
\end{aligned}
$$

According to [3]

$$
\mathcal{P}\left\{k \in \mathcal{R}: \overline{\mathbf{h}}_{k} \in \bigcup_{i=1}^{N_{t}} \mathcal{B}_{\epsilon}\left(\phi_{i}\right)\right\} \leq N_{t} \epsilon^{N_{t}-1}
$$

Then we have obtain:

$$
K_{\text {avg }} \leq K N_{t} \mathcal{P}\left\{\left\|\mathbf{h}_{k}\right\|^{2}>\gamma_{t h}\right\} \epsilon^{N_{t}-1}
$$

The set $\mathcal{T}_{1}$ is determined by the incomplete gamma distribution $\operatorname{Gamma}\left(N_{t}, 1\right)$ which can be bounded by [4][5]:

$$
\left[1-e^{-\beta \gamma}\right]^{N_{t}} \leq \int_{0}^{\gamma_{t h}} f_{\gamma}(\gamma) d \gamma \leq\left[1-e^{-\gamma}\right]^{N_{t}}
$$

where $\beta=\left(N_{t} !\right)^{-\frac{1}{N_{t}}}$ and $f_{\gamma}(\gamma)$ is the probability density function $\chi_{2}^{2}\left(N_{t}\right)$.

In Figure 1 we present the curves of the pair $\left(\gamma_{t h}, \epsilon_{t h}\right)$ allowing predetermined probability $\mathcal{P}\left\{k \in \mathcal{T}_{3}\right\}$ (10\% to $50 \%$ ). For each probability, it will be necessary to choose a pair by privileging either the criterion on the norm or the criterion on orthogonality.

\section{FEEDBACK LINK}

\subsection{Quantized feedback link}

To address the lack of perfect CSIT, a classical solution is to quantize CDI and CQI before transmission over the finite rate feedback link. In [6], the Lloyd algorithm was suggested for the design of the beamforming vector codebook. The codebook should be constructed by minimizing the maximum inner product between codewords and this results in the Grassmannian line packing solution when the channel vector is i.i.d.

In the quantized feedback scheme, the precoding vector $\mathbf{w}$ is taken from a set of $2^{B}$ vectors where $B$ is the number of feedback bits.

In contrast to the normalized i.i.d. channel isotropically distributed in $\mathcal{O}^{N_{t}}$, an important aspect of a limited feedback codebook tailored to a spherical cap region is the quantization of the 


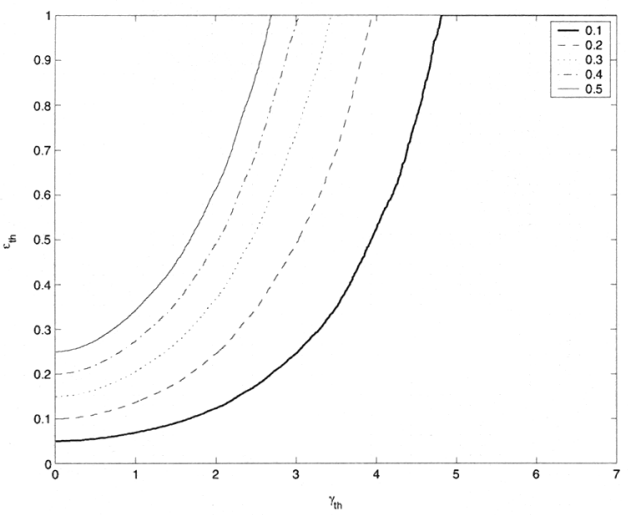

Figure 1: $\epsilon_{t h}$ and $\gamma_{t h}$ for $\mathcal{P}\left\{k \in \mathcal{T}_{3}\right\}=0.1$ to 0.5

localized region or local packing. A local Grassmannian packing with parameters $N_{t}, N, \mathbf{0}, \epsilon_{t h}$ is a set of $N$ vectors, where $N$ is the codebook size, $\mathbf{w}_{i}, i=1, \ldots, N$, constrained to a spherical cap $\mathcal{B}_{\epsilon}(\mathbf{o})$ in $\mathcal{O}^{N_{t}}$ such that

$$
\min _{1 \leq i<j \leq N} d^{2}\left(\mathbf{w}_{i}, \mathbf{w}_{j}\right)
$$

is maximized.

From the spherical cap $\mathcal{B}_{\epsilon}(\mathbf{0})$ it is possible to compute the rotated spherical cap $\mathcal{B}_{\epsilon}\left(\mathbf{o}_{\text {rot }}\right)$ by applying the following rotation map:

$$
r(\mathbf{o})=\mathbf{U}_{\text {rot }} \mathbf{o} \triangleq \mathbf{o}_{\text {rot }}
$$

where $\mathbf{U}_{\text {rot }}$ is the unitary rotation matrix [7]

As in the i.i.d. case, we use vector quantization to design these local packings. For the $\mathcal{T}_{2}$ and $\mathcal{T}_{3}$ criteria, the codebook must be adapted according to the orthogonal vectors $\phi_{i}$. From the local packing, it is possible to compute the local packing associated to a rotation using the rotation matrix. When the user CDI is inside the spherical cap region, the user will feedback $\log _{2}(N)$ bits corresponding to the codebook index. In addition to that, it will be necessary to feedback $\log _{2}\left(N_{t}\right)$ bits corresponding to the index of the vector $\phi_{i}$. Consequently, for a codebook size $N$, $B=\log _{2}\left(N \times N_{t}\right)$ bits will be necessary to quantify the CDI.

\subsection{Analog feedback link}

In analog feedback [8] [9] [10], the channel coefficients are explicitly transmitted on the uplink using unquantized quadratureamplitude modulation. Since the dimension of the channel vectors is $N_{t}, N_{t}$ channel use will be used to send the channel coefficients.

A simplifying assumption is that we consider no fading and orthogonal access in the feedback link. The signal is corrupted by AWGN.

Each selected user $k \in \mathcal{T}_{3}$ feedbacks its channel information to the transmitter through the noisy uplink channel. The received vector $g_{k}$ of dimension $N_{t} \times 1$ from the $k$-th user at the base station is:

$$
\begin{aligned}
\mathbf{g}_{k} & =\beta \mathbf{h}_{k}+\mathbf{b}_{k}, \quad k \in \mathcal{T}_{3} \\
& =\left\|\mathbf{g}_{k}\right\| \overline{\mathbf{g}}_{k}
\end{aligned}
$$

where $\beta=\sqrt{ } \bar{P}$ where $\bar{P}$ is the average transmit power, $\mathbf{b}_{k} \in$ $\mathcal{C}^{N_{t} \times 1}$ is the i.i.d zero mean Gaussian noise added to the uplink channel.

When using the $\mathcal{T}_{1}$ criterion, the received signal at the base station is directly exploited to calculate the sum rate, select the users and perform the precoding. However, when using the $\mathcal{T}_{3}$ criterion it is possible to improve the performance of the system by exploiting the semi-orthogonal constraint to suppress part of the uplink noise. The normalized estimated channel at the base station, $\hat{\overline{\mathbf{h}}}_{k}$ should lie in the spherical cap described by the square radius $\epsilon_{t h}$. In this paper, we propose two solutions to exploit this property.

The proposed algorithm:

Firstly, we check the condition as below:

$$
\min _{i=1, \ldots, N_{t}}\left(1-\left|\overline{\mathbf{g}}_{k}{ }^{H} \phi_{i}\right|^{2}\right) \leq \epsilon_{t h}
$$

If the condition is satisfied, in order to obtain precoding vector, we assign $\hat{\overline{\mathbf{h}}}_{k} \longleftarrow \overline{\mathbf{g}}_{k}$.

Otherwise, we apply the following algorithm:

- Option 1: Since $\overline{\mathrm{g}}_{k} \notin \bigcup_{i=1}^{N_{t}} \mathcal{B}_{\epsilon}\left(\phi_{i}\right)$ we first search the closest vector $\phi_{i}$ from $\overline{\mathbf{g}}_{k}$

$$
\text { - j= } \underset{i=1, \ldots, N_{t}}{\arg \min }\left(1-\left|\overline{\mathbf{g}}_{k}^{H} \phi_{i}\right|^{2}\right)
$$

- Find $\hat{\overline{\mathbf{h}}}_{k}$ such as

$$
\begin{array}{cc}
\min \left\|\overline{\mathbf{g}}_{k}-\hat{\overline{\mathbf{h}}}_{k}\right\|^{2} \\
\text { subject to } & 1-\left|\hat{\overline{\mathbf{h}}}_{k}^{H} \phi_{j}\right|^{2}=\epsilon_{t h}
\end{array}
$$

- $\hat{\overline{\mathbf{h}}}_{k}$ is the closest vector from $\overline{\mathbf{g}}_{k}$ and situated at the surface of the spherical cap $\mathcal{B}_{\epsilon}\left(\phi_{j}\right)$.

- Since $\left\|\hat{\overline{\mathbf{h}}}_{k}\right\|^{2}=1$ This optimization can be simplified as:

$$
\begin{array}{ll} 
& \min \left(-\hat{\overline{\mathbf{h}}}_{k}^{H} \overline{\mathbf{g}}_{k}\right) \\
\text { subject to } & 1-\left|\hat{\overline{\mathbf{h}}}_{k}^{H} \phi_{j}\right|^{2}=\epsilon_{t h}
\end{array}
$$

- Equations (20) and (21) correspond to a SOCP problem (linear programming with quadratic constraint). In order to solve this problem, MATLAB routines from optimization toolbox or special software for SOCP can be applied.

- Option 2: A simpler solution consists in associating the closest orthogonal vector to $\hat{\overline{\mathbf{h}}}_{k}$ :

$$
\hat{\overline{\mathbf{h}}}_{k}=\phi_{j}
$$

Then, we assign $\hat{\mathbf{h}}_{k}=\hat{\overline{\mathbf{h}}}_{k}\left\|\mathbf{g}_{k}\right\| / \beta$.

\section{SIMULATION RESULTS}

We consider $N_{t}=2$ antennas at the base station. $\gamma_{t h}$ for criterion $\mathcal{T}_{1}$ and the pair $\left(\gamma_{t h}, \epsilon_{t h}\right)$ for criterion $\mathcal{T}_{3}$ are theoretically calculated in order to have an average number of users in the cell $K_{a v g}=4$. Only these users feedback their $Q$ bits for the quantization of each channel gain $\left\|\mathbf{h}_{k}\right\|^{2}$ and $B$ bits corresponding to the codebook index of their quantized CDI. Exploiting this feedback 
information, the base station will select the $N_{t}$ users in order to maximize the sum data rate.

In all our simulations we take the case where $K_{a v g}=4$ and so $\gamma_{t h}$ and the pair $\left(\gamma_{t h}, \epsilon_{t h}\right)$ should be chosen such as $\gamma_{t h}=$ $\left[\begin{array}{llllll}0 & 1.7 & 3 & 4 & 5 & 5.8\end{array}\right]$ for criterion $\mathcal{T}_{1}$, and the pair $\left(\gamma_{t h}, \epsilon_{t h}\right)=$ $[(0,1)(1,0.35)(2,0.23)(2.5,0.18)(3,0.1)(3.8,0.09)]$ for criterion $\mathcal{T}_{3}$.

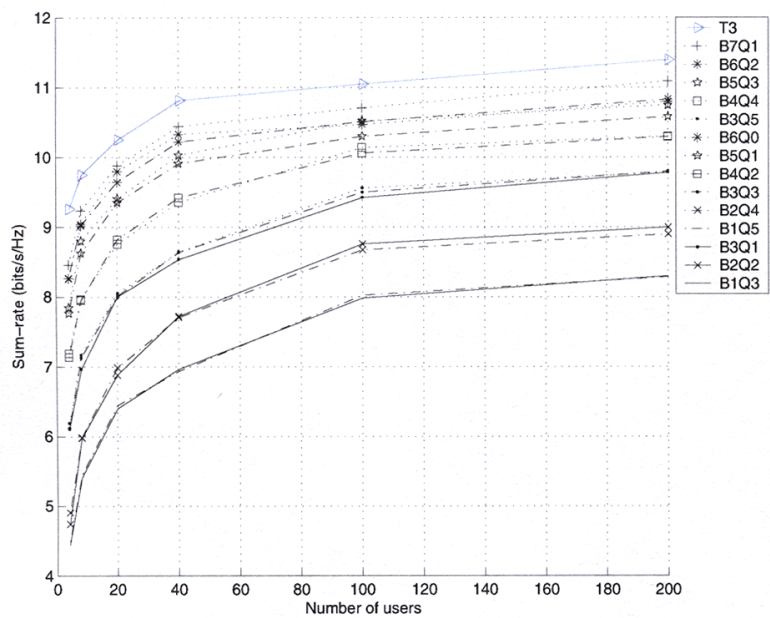

Figure 2: Sum rate versus the number of users for Criterion $\mathcal{T}_{3}$ under $N_{t}=2, S N R=15 d B, K_{a v g}=4$ users and various feedback bits.

In Figure 2 we give the sum rate performances of $\mathcal{T}_{3}$ schemes at $S N R=15 d B$, for different numbers of active users in the cell and number of feedback bits $F=B+Q$ per user. For $F=4,6$ and 8 bits, we modify the number of CDI bits (B) and CQI bits (Q). As shown in this Figure, the performances are almost independent of the number of CQI bits. Surprisingly, the sum-rate performance is not affected when there is no feedback information about the channel norm $(Q=0)$ of the selected users. On the contrary, the number of CDI bits has a huge impact on the performance.

Criterion $\mathcal{T}_{1}$ outperforms criterion $\mathcal{T}_{3}$ in the case of $F=8 \mathrm{bits}$, but the two criteria have slightly the same sum-rate performance for $F=6$ feedback bits. For the case of less feedback bits such as $F=4$, not only does the $\mathcal{T}_{3}$ sum-rate performance becomes better than the $\mathcal{T}_{1}$ performance but also the $\mathcal{T}_{1}$ degradation is larger relative to that of $\mathcal{T}_{3}$. This is due to a smaller vector quantization error for the $\mathcal{T}_{3}$ criterion since all the codebook vectors are lying in the spherical cap described by the square radius $\left(\epsilon_{t h}\right)$.

In Figure 3 we present the sum-rate versus the average SNR for the system with $K=100, F=4,7$ bits such as $B=7$ $Q=0$ and $B=4 Q=0$. We also plot random beamforming (RBF), $\mathcal{T}_{1}$ and $\mathcal{T}_{3}$ performance curves without quantization. It is known that RBF performs well in a system with a large number of users. However, in the case of $F=8$ bits the $\mathcal{T}_{1}$ and $\mathcal{T}_{3}$ criteria outperform the RBF scheme with reduced feedback. For the case of $F=7 \mathrm{bits}$, the $\mathcal{T}_{3}$ criterion outperforms the $\mathcal{T}_{1}$ criterion at high SNR when the system becomes sensitive to the interference and the users' orthogonality is more critical. For the case of $F=4$ bits, criterion $\mathcal{T}_{3}$ always outperforms criterion $\mathcal{T}_{1}$. The performance gain increases with SNR.

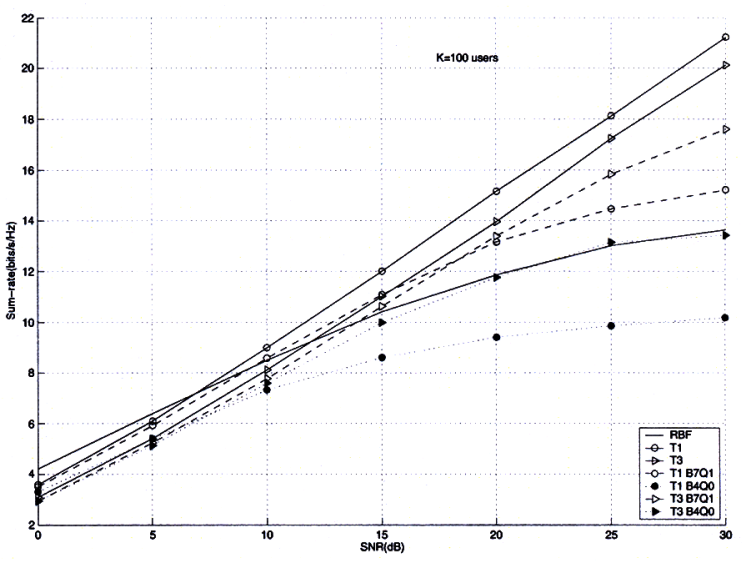

Figure 3: Sum rate versus snr for RBF, Criteria $\mathcal{T}_{1}$ and $\mathcal{T}_{3}$ under $N_{t}=2, K=100$ and 4,8 bits.

In order to fairly compare the different criteria when the feedback link is noisy, we have fixed the same average transmit energy per symbol for both analog and quantized feedback link.

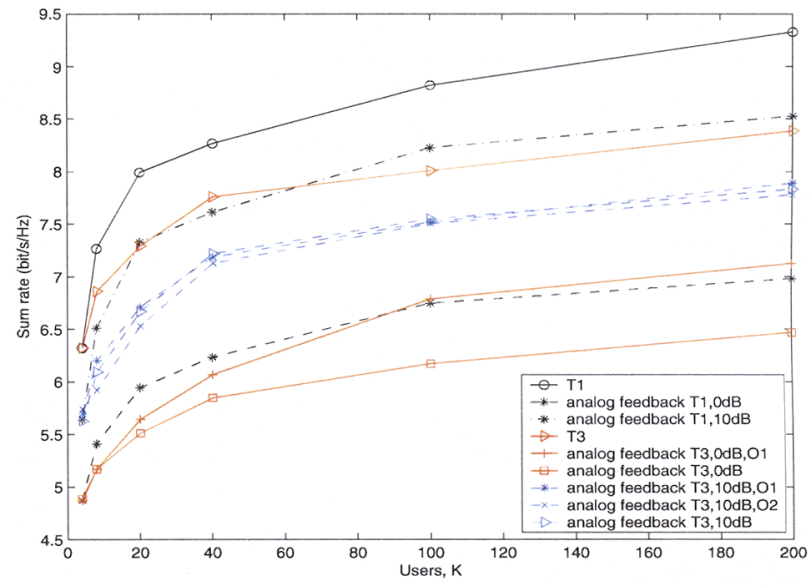

Figure 4: Sum rate versus number of users for $\mathcal{T}_{3}$ with the proposed algorithm for $N_{t}=2$ in noisy feedback channel with different SNR values.

In Figure 4, we evaluate the performance of criterion $\mathrm{T} 3$ with the different proposed options at different $\left(E_{S} / N_{0}\right)_{U L}$. If the SNR is high (10dB), the improvement is limited; however, at low SNR, performance of the $\mathcal{T}_{3}$ criterion can be increased using option 1 . In that case, the $\mathcal{T}_{3}$ criterion outperforms the $\mathcal{T}_{1}$ criterion.

In Figures 5 and 6 we compare the performance of the analog and quantized feedback links for $\left(E_{S} / N_{0}\right)_{U L}=10$ and $0 \mathrm{~dB}$ respectively. For all the schemes, 2 symbols are needed to transmit analog channel or the codeword index. The modulation is 16QAM when the number of bits is $F=8$ whereas the modulation is QPSK when $F=4$.

According to the simulation results, the analog feedback for criterion $\mathcal{T}_{1}$ gives a better performance than criterion $\mathcal{T}_{3}$ at high 


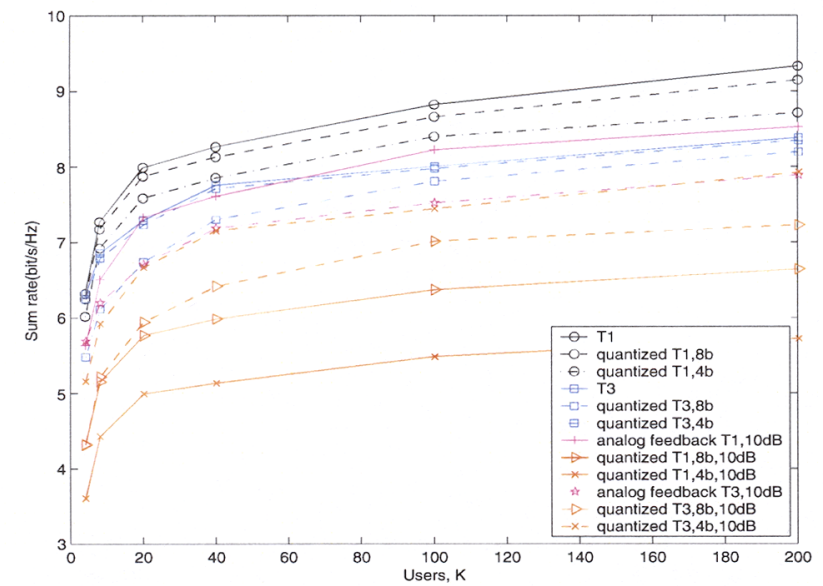

Figure 5: Sum rate versus number of users for Criteria $\mathcal{T}_{1}$ and $\mathcal{T}_{3}$ for $N_{t}=2$ in noisy feedback channel with $S N R_{U L}=10 \mathrm{~dB}$.

SNR. However, when the uplink noise variance increases, the performance of the $\mathcal{T}_{3}$ criterion with analog feedback gives the same result as the $\mathcal{T}_{1}$ criterion. Moreover, the $\mathcal{T}_{3}$ criterion with 4 bits quantized feedback gives slightly better results at low SNR than analog feedback.

\section{CONCLUSION}

In this paper we have evaluated the performance of user selection criteria based on norm and semi-orthogonality of the channels considering both analog and quantized CDI and CQI feedback links. We have shown that, when the codebook is designed according to local regions, the quantized version of the proposed criterion $\mathcal{T}_{3}$ outperforms the norm-only criterion $\mathcal{T}_{1}$. Since both criteria perform a selection of users using the norm of their channels, the number of quantized CQI has almost no impact on the performances and only CDI information is important at the transmitter to maximize the sum-rate performance. Then we have considered noisy feedback channels. We have shown that for analog feedback it is possible to improve the performance of criterion $\mathcal{T}_{3}$ by exploiting the semi-orthogonal constraint. For the noisy quantized CDI feedback link, we have shown that criterion $\mathcal{T}_{1}$ for the same number of symbols per codeword, is much more sensitive to uplink noise than criterion $\mathcal{T}_{3}$. This sensibility increases when we decrease the number of codewords. Compared to the point to point MISO system, when using a semi-orthogonal criterion in multiuser MISO system, the performance of quantized feedback link with few bits can be very close to that of analog feedback link.

\section{REFERENCES}

[1] D. Gesbert and M. -S. Alouini, "How much feedback is multi-user diversity really worth", In Proceedings of IEEE Intern. Conf. On Communications (ICC)”, , 2004.

[2] T. Yoo and A. Goldsmith, "On the optimality of multiantenna broadcast scheduling using zero-forcing beamforming", IEEE Journal on Sel. Areas in Commun. (JSAC), vol. 24, no. 3, pp.528-541, Mar. 2006.

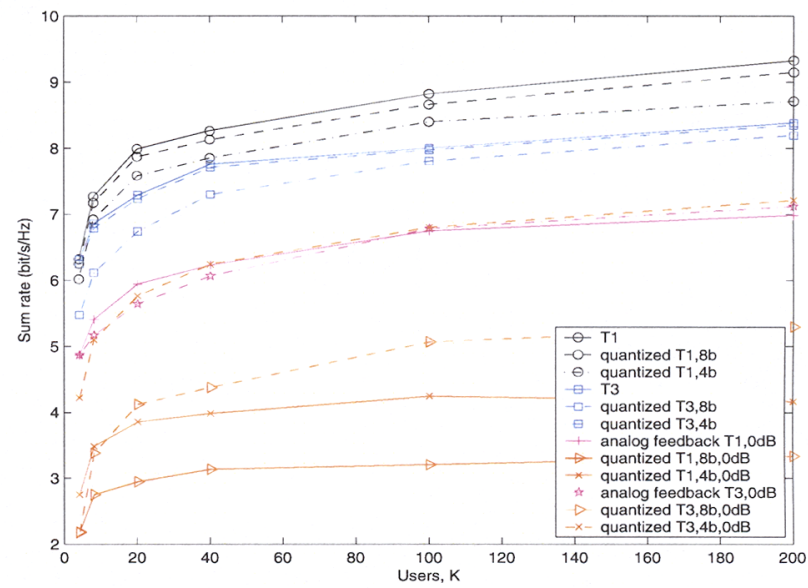

Figure 6: Sum rate versus number of users for Criteria $\mathcal{T}_{1}$ and $\mathcal{T}_{3}$ for $N_{t}=2$ in noisy feedback channel with $S N R_{U L}=0 d B$.

[3] K. K. Mukkavilli, A. Sabharwal, E. Erkip, B. Aazhang, "On beamforming with finite rate feedback in multiple antenna systems", IEEE Trans. on Information Theory, vol. 49, pp.2562-2579", Oct. 2003.

[4] M. Sharif , B. Hassibi, "On the capacity of MIMO Broadcast channels with partial side information", IEEE Trans. on Information Theory, vol. 51, pp.506-522”, Sept. 2005.

[5] K. Huang, R. W. Heath, J. Andrews, "Multi-user aware limited feedback for MIMO systems", submitted to IEEE Trans. on on Signal Processing, Jan. 2007.

[6] A. Narula, M. J. Lopez, M. D. Trott, G. W. Wornell, "Efficient use of side information in multiple antenna data transmission over fading channels", IEEE Journal on Sel. Areas in Commun. (JSAC), pp.1423-1436, Oct. 1998.

[7] V. Raghavan, R. W. Heath, Jr., A. M. Sayeed, "Systematic Codebook Designs for Quantized Beamforming in Correlated MIMO Channels", IEEE Journal on Sel. Areas in Commun. (JSAC), vol. 25, pp.1298-1310”, Sept. 1998.

[8] M. Gaspar, R. Rimoldi, M. Vetterli, "To code, or not to code: lossy source channel communication revisited" in IEEE Trans. on Information Theory, vol. 49, pp.1147-1158, May 2003.

[9] G. Caire, N. Jindal, M. Kobayashi, and N. Ravindran, "Quantized vs. Analog Feedback for the MIMO Downlink: A Comparison between Zero-Forcing Based Achievable Rates", IEEE International Symposium on Information Theory (ISIT), June 2007

[10] D. Le Ruyet and Berna Özbek, "Partial and Analog Feedback for MISO Precoding Systems", In Proceedings of IEEE Intern. Conf. On Communications (ICC), Glasgow, UK, 2007. 